\title{
A Survey of Pregnancy Outcomes for Women of Childbearing Age with Inflammatory Bowel Disease in South China
}

\author{
Miao Li1,2*, Qingfan Yang1,2*, Qian Cao ${ }^{3}$, Jian Tang1,2, Min Zhi1,2, Kang Chao',2, Lingjie Huang3, \\ Yuan Zhao ${ }^{3}$, Pinjin $\mathrm{Hu}^{1,2}$, Xiang Gao ${ }^{1,2 \#}$ \\ ${ }^{1}$ Department of Gastroenterology, The Sixth Affiliated Hospital of Sun Yat-sen University, Guangzhou, China \\ ${ }^{2}$ Guangdong Provincial Key Laboratory of Colorectal and Pelvic Floor Diseases, The Sixth Affiliated Hospital, Sun Yat-sen \\ University, Guangzhou, China \\ ${ }^{3}$ Department of Gastroenterology, Sir Run Run Shaw Hospital, School of Medicine, Zhejiang University, Hangzhou, China \\ Email: "helengao818@163.com
}

How to cite this paper: Li, M., Yang, Q.F., Cao, Q., Tang, J., Zhi, M., Chao, K., Huang, L.J., Zhao, Y., Hu, P.J. and Gao, X. (2018) A Survey of Pregnancy Outcomes for Women of Childbearing Age with Inflammatory Bowel Disease in South China. Open Journal of Gastroenterology, 8, 348-361.

https://doi.org/10.4236/ojgas.2018.810037

Received: October 3, 2018

Accepted: October 14, 2018

Published: October 17, 2018

Copyright $(9) 2018$ by authors and Scientific Research Publishing Inc. This work is licensed under the Creative Commons Attribution International License (CC BY 4.0).

http://creativecommons.org/licenses/by/4.0/

\begin{abstract}
Purpose: Women of childbearing age with inflammatory bowel disease (IBD) in South China were studied to understand the effects of IBD on pregnancy outcomes. Materials and Methods: One hundred thirty-nine women treated at two large IBD centers participated. Pregnancy outcome data were collected by telephone interviews. Results: Ninety patients (90/139; 64.7\%) did not pursue pregnancy after IBD diagnosis because of concerns regarding adverse effects. Fifteen Crohn's disease (CD) and 12 ulcerative colitis (UC) patients pursued pregnancy and achieved successful conception. Twenty-five patients were treated with drugs before pregnancy; however, 15 (15/25; 60\%) discontinued the use of all drugs 3 months before pregnancy. The other 10 patients continued mesalazine and one continued azathioprine during pregnancy. Four CD and five UC patients had mild disease at conception; the others were in remission. Two CD patients and one UC patient experienced mild disease relapse during pregnancy. They refused all drugs except mesalazine. One UC patient with mild disease during pregnancy had pregnancy-induced hypertension. All newborns were healthy. There was no difference between pregnancy outcomes of $\mathrm{CD}$ and UC patients $(\mathrm{P}>0.05)$. No relationship was found between disease activity and pregnancy outcomes $(P>0.05)$. Patients with active disease during pregnancy tended to choose cesarean delivery, especially in $C D$ patients with active disease $(P=0.028)$. Conclusion: Many female IBD patients may not pursue pregnancy because of concerns regarding adverse effects on the pregnancy. Patients were more willing to conceive
\end{abstract}

^These authors contributed equally to this work and were considered to be co-first authors. 
during disease remission. Some refused all IBD medications except mesalazine. Relapse was uncommon or mild during pregnancy. Disease activity had an obvious effect on the delivery mode of $\mathrm{CD}$ patients.

\section{Keywords}

Pregnancy, Cesarean, Inflammatory Bowel Disease

\section{Introduction}

Inflammatory bowel disease (IBD), including ulcerative colitis (UC) and Crohn's disease (CD), is a chronic non-specific inflammatory disease with unknown etiology that widely involves the gastrointestinal tract. IBD onset is usually noted in young adults with a prolonged and recurrent disease course, and it severely affects all aspects of patients' lives [1]. Childbirth is very important for some women of childbearing age. Many female IBD patients have fertility concerns when diagnosed with IBD. However, the effects of IBD on childbirth are still unclear. Therefore, it is important to investigate whether IBD and its activity, medication, and surgical treatment would affect the fertility of female IBD patients.

Some studies have shown that the presence of IBD increases the risk of various pregnancy complications, such as miscarriage, premature delivery, low-birth-weight infants, and cesarean delivery [2] [3] [4] [5] [6]. However, some studies found that there is no significant difference in the fertility process and outcomes between the normal population and patients with CD and UC [4]. Moreover, the medication used to treat pregnant patients with IBD is controversial and remains unclear. However, the participants in those studies were primarily Caucasian, and only a few studies were about the fertility of Asian women with IBD. Because environmental and genetic factors play an important role in the onset and development of IBD, and because there may be differences in the fertility of patients in different populations, it is necessary to further investigate the fertility of Asian IBD patients. This study conducted a survey of the fertility of women of childbearing age with IBD in South China to understand the effects of IBD on fertility and delivery outcomes.

\section{Materials and Methods}

\subsection{Study Participants}

This retrospective study was approved by the ethics committee of the Sixth Affiliated Hospital of Sun Yat-sen University. The inclusion criteria of patients was: 1) Meet the diagnosis of IBD; 2) Female patients of childbearing age (range, 20 49 years old) who were recommended by China's family planning policy and were followed up in the Sixth Affiliated Hospital of Sun Yat-sen University or the Sir Run Run Shaw Hospital of Zhejiang University treatment centers from April 2014 to August 2017; 3) Patients agreed to accept the pregnancy survey by 
telephone interview. We excluded patients who had ever been diagnosed with malignant tumors and immunosuppressive diseases such as human immunodeficiency virus (HIV), rheumatoid arthritis (RA), or systemic lupus erythematosus (SLE). Patients refused to answer our investigation questions also been excluded.

The diagnosis of IBD was confirmed according to the Lennard-Jones criteria [7]. The clinical diagnosis was established on the basis of clinical symptoms, physical examination, colonoscopy, results of intestinal imaging, histology, and surgical specimens. Stool test and parasites detecting were performed to exclude enteric infections. Tuberculin skin test, T-spot, chest X-ray, and colonoscopy with biopsy for acid-fast bacilli staining were performed to exclude intestinal tuberculosis. Ischemia, non-steroidal anti-inflammatory drug induced ulceration, and radiation colitis were also excluded by medical history. Patients who were only clinically diagnosed of IBD and without the support of histology were followed up for at least one year to confirm their diagnosis. Patients' demographic data and clinical classification characteristics, such as sex, disease course, symptoms, smoking history, and family history, were collected. The clinical classification was assessed according to the Montreal classification of IBD [8].

\subsection{Fertility Survey}

A questionnaire was designed for this survey. The content included the course of the disease, marital status, number of children, bowel resection history, perianal disease history, premorbid fertility status, and desire for fertility after the onset of IBD. Patients who became pregnant after IBD onset were interviewed about the details of their IBD medications before and after pregnancy, disease activity, pregnancy complications, delivery outcomes (miscarriage or premature delivery), mode of delivery (cesarean delivery or vaginal delivery), birth weight, and congenital malformations. Telephone follow-up interviews were conducted based on the content of the questionnaire.

\subsection{Relevant Definitions}

During the $\mathrm{CD}$ activity period, the $\mathrm{CD}$ activity index (CDAI) was calculated, and CDAI $>150$ was defined as active CD. Mild UC disease activity was defined as diarrhea less than four times per day, with slight or no blood in the stool, no fever or rapid pulse, slight or no anemia, and normal erythrocyte sedimentation rate (ESR). Severe UC severe disease was defined as diarrhea more than six times per day, obvious mucopurulent bloody stool, body temperature $>37.5^{\circ} \mathrm{C}$, pulse $>90$ beats/minute, hemoglobin $<100 \mathrm{~g} / \mathrm{L}$, and ESR $>20 \mathrm{~mm} / \mathrm{h}$. Moderate UC disease activity was more harsh than mild UC but less harsh than severe UC [9]. Miscarriage was defined as inadvertent pregnancy termination before 28 weeks and fetal weight $<1000 \mathrm{~g}$. Premature delivery was defined as childbirth at 28 weeks or later but before 37 weeks; these newborns were referred to as premature infants. A small-for-gestational-age infant was defined as a newborn with body weight below the 10th percentile for newborns of the same gestational 
age [2]. Adverse pregnancy outcomes referred to the presence of one or more of the following complications and scenarios: miscarriage, premature delivery, preeclampsia, small for gestational age status, Apgar score less than 5 points, or a newborn that required care in the intensive care unit.

\subsection{Statistical Analyses}

All results are expressed as mean \pm standard deviation or frequency. Differences were compared using the Chi-square test or Fisher's exact test. All statistical analyses were performed using statistical software SPSS 13.0, and $\mathrm{P}<0.05$ indicated a statistically significant difference.

\section{Results}

\subsection{Basic Information of the Study Participants}

A total of 166 women of childbearing age with IBD were recruited to participate in telephone follow-up interviews with the IBD clinic centers of the Sixth Affiliated Hospital of Sun Yat-sen University or the Sir Run Run Shaw Hospital of Zhejiang University treatment centers during April 2014 to August 2017. Nine $\mathrm{CD}$ and $18 \mathrm{UC}$ patients did not respond to or rejected the telephone follow-up interviews; therefore, a total of 139 women ( $82 \mathrm{CD}$ and 57 UC patients) of childbearing age with IBD participated and were included in this study. Of the 139 patients enrolled, the average age was 35.25 years old. The average disease course of CD was $4.75 \pm 4.25$ years and UC was $4.35 \pm 3.2$ years. All patients had no family history of IBD and only 2 patients had smoking history. Four patients of $\mathrm{CD}$ experienced bowel resection surgery and 7 patients with $\mathrm{CD}$ had perianal disease. 12 patients experienced mild disease activity before or during pregnancy with symptoms such as abdominal pain, diarrhea, fever or blood stool. Basic demographic information of the patients is detailed in Table 1.

\subsection{Survey Regarding the Desire for Fertility for Women with IBD}

Before the IBD diagnosis, the included patients had given birth to 108 infants; however, they only gave birth to 27 infants after being diagnosed with IBD. A total of 90 patients (90/139; 64.7\%; $53 \mathrm{CD}$ and 37 UC patients) decided to no longer pursue pregnancy. Of the patients who were followed-up by our hospital, 38 decided to no longer pursue pregnancy after IBD diagnosis. When asked for more details regarding the specific reasons, they all expressed that they did not have any fertility abnormalities. Ten CD and 19 UC patients decided not to conceive again because they had already given birth and were in accordance with the national family planning policy. The other nine patients $(9 / 38 ; 23.7 \%)$ decide to no longer pursue pregnancy because they were concerned about the possible adverse effects of IBD or its medication on the fetus (Table 2, Figure 1).

\subsection{Medications before and after Pregnancy}

Thirty IBD patients (17 CD and 13 UC patients) were thought to have become 
Table 1. Basic characteristics of the IBD patients.

\begin{tabular}{|c|c|c|}
\hline Clinical features & $\mathrm{CD}(\mathrm{n}=82)$ & $\mathrm{UC}(\mathrm{n}=57)$ \\
\hline Course of disease (year) & $4.75 \pm 4.25$ & $4.35 \pm 3.2$ \\
\hline Family history (yes) & 0 & 0 \\
\hline $\begin{array}{c}\text { Smoking history } \\
\text { Yes } \\
\text { No }\end{array}$ & $\begin{array}{c}1(1.2 \%) \\
81(98.8 \%)\end{array}$ & $\begin{array}{c}1(1.8 \%) \\
56(98.2 \%)\end{array}$ \\
\hline $\begin{array}{c}\text { Intestinal surgery history } \\
\text { Yes } \\
\text { No }\end{array}$ & $\begin{array}{c}4(4.9 \%) \\
78(95.1 \%)\end{array}$ & $\begin{array}{c}0(\%) \\
57(100 \%)\end{array}$ \\
\hline \multicolumn{3}{|l|}{ Disease location (CD) } \\
\hline L1 (ileum) & $4(4.9 \%)$ & - \\
\hline L2 (colon) & $12(14.6 \%)$ & - \\
\hline L3 (ileocolon) & $49(59.8 \%)$ & - \\
\hline L4 (upper gastrointestinal involvement) & $17(20.7 \%)$ & - \\
\hline \multicolumn{3}{|l|}{ Disease location (UC) } \\
\hline E1 (rectum) & - & $14(24.6 \%)$ \\
\hline E2 (left semicolon) & - & $17(29.8 \%)$ \\
\hline E3 (whole colon) & - & $26(45.6 \%)$ \\
\hline \multicolumn{3}{|l|}{ Disease behavior (CD) } \\
\hline B1 (non-stricture, non-penetrating) & $51(62.2 \%)$ & - \\
\hline B2 (stricture) & $18(22.1 \%)$ & - \\
\hline B3 (penetrating) & $13(15.7 \%)$ & - \\
\hline \multicolumn{3}{|l|}{ Perianal lesions } \\
\hline Yes & $7(8.5 \%)$ & 0 \\
\hline No & $75(91.5 \%)$ & 0 \\
\hline
\end{tabular}

IBD, inflammatory bowel disease; $\mathrm{CD}$, Crohn's disease; UC, ulcerative colitis.

Table 2. Survey of the desire to pursue pregnancy after IBD diagnosis.

\begin{tabular}{ccc}
\hline Desire after diagnosis & CD $(\mathbf{n})$ & UC (n) \\
\hline Yes & 29 & 20 \\
No & 53 & 37
\end{tabular}

IBD, inflammatory bowel disease; CD, Crohn's disease; UC, ulcerative colitis.

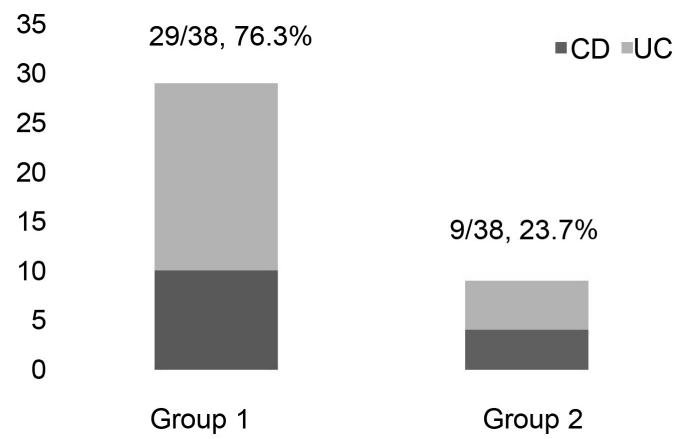

Figure 1. Survey of the reasons why patients stopped pursuing pregnancy after IBD diagnosis (Data from the IBD Diagnosis and Treatment Center of the Sixth Affiliated Hospital of Sun Yat-sen University). Group 1: Patients stopped pursuing pregnancy because they had already given birth before being diagnosed with IBD and also were in accordance with national family planning policy; Group 2: Patients stopped pursuing pregnancy because they were worried about the adverse effects of IBD and its medication on the fetus. 
pregnant after the IBD diagnosis; however, among them, two $\mathrm{CD}$ patients and one UC patient became pregnant before the IBD diagnosis was confirmed. The two $C D$ patients had been treated with infliximab andazathioprine. Infliximab was used continuously throughout the pregnancy, but azathioprine was discontinued before the pregnancy. The UC patient was treated with mesalazine before and after pregnancy. Twenty seven patients became pregnant after the IBD diagnosis. Among them, except for 2 UC patients, 25 patients used drug maintenance treatment prior to the pregnancy, including the use of salazopyrin, mesalazine, glucocorticoids, azathioprine/mercaptopurine, infliximab, and methotrexate. Fifteen patients $(15 / 25 ; 60 \%)$ discontinued the drugs 3 months before conception. The exact drug histories before and after pregnancy are listed in Table 3. Of the $15 \mathrm{CD}$ patients who conceived after the diagnosis, only two underwent azathioprine therapy before pregnancy and discontinued the drug 3 to 6 months before conception. One was treated with azathioprine and mesalazine but discontinued azathioprine before conception. One was treated with infliximab and azathioprine but discontinued infliximab before conception; however, she continued azathioprine during pregnancy. During the pregnancy, her disease activity

Table 3. Drug administration for patients with successful conception after IBD diagnosis.

\begin{tabular}{|c|c|c|}
\hline Drugs & $\mathrm{CD}(\mathrm{n})$ & $\mathrm{UC}(\mathrm{n})$ \\
\hline Salazopyrin & 1 & 2 \\
\hline Discontinuation before pregnancy & 1 & 2 \\
\hline Continuous administration during pregnancy & 0 & 0 \\
\hline Mesalazine & 7 & 8 \\
\hline Discontinuation before pregnancy & 3 & 2 \\
\hline Continuous administration during pregnancy & 4 & 6 \\
\hline Hormones & 2 & 0 \\
\hline Discontinuation before pregnancy & 2 & 0 \\
\hline Continuous administration during pregnancy & 0 & 0 \\
\hline Azathioprine/mercaptopurine & 4 & 0 \\
\hline Discontinuation before pregnancy & 3 & 0 \\
\hline Continuous administration during pregnancy & 1 & 0 \\
\hline Methotrexate & 1 & 0 \\
\hline Discontinuation before pregnancy & 1 & 0 \\
\hline Continuous administration during pregnancy & 0 & 0 \\
\hline Infliximab & 2 & 0 \\
\hline Discontinuation before pregnancy & 2 & 0 \\
\hline Continuous administration during pregnancy & 0 & 0 \\
\hline No medication before pregnancy & 0 & 1 \\
\hline $\begin{array}{l}\text { No medication before pregnancy but used } \\
\text { medication after conception }\end{array}$ & 0 & 1 \\
\hline
\end{tabular}

${ }^{*}$ The exact drug histories before and after pregnancy of CD and UC patients. There was no significant difference of the drug histories of the two groups. 
recurred, but she refused the medication. One CD patient was treated with infliximab and used probiotics for treatment; however, she discontinued infliximab before conception and then discontinued probiotics throughout the pregnancy (Table 3). Patients were more willing to discontinue their medications such as immunosuppressant and biological drugs after pregnancy and some patients could accept continuing using mesalazine after pregnancy.

\subsection{Pregnancy Outcomes and Disease Activity}

1) Disease activity before and after pregnancy: Of the 27 patients who successfully gave birth, 18 (67\%) had disease remission during pregnancy. Of the 15 CD patients, 11 had disease remission before pregnancy and four had mild disease activity, including two who had disease remission during early pregnancy and two who refused medication because of only mild disease activity. Among the 12 UC patients, seven had disease remission before pregnancy and five who had mild disease activity refused to use any drugs other than mesalazine. During the pregnancy, two CD patients who had disease remission experienced recurrence but refused medication; one UC patient presented with mild disease recurrence and used mesalazine treatment.

2) Pregnancy outcomes: Of the 30 patients who were suspected to be pregnant after IBD onset, three conceived before the confirmation of IBD and did not experience disease recurrence or complications. Of the $15 \mathrm{CD}$ patients who successfully gave birth, eight had a vaginal delivery and seven had a cesarean delivery; none of these patients had pregnancy complications such as miscarriage, premature delivery, preeclampsia and small for gestational age status, et al. All of them delivered healthy and full-term newborns. None of the babies got Apgar score less than 5 points. Of the 12 UC patients who successfully gave birth, seven had a vaginal delivery and five had a cesarean delivery. One patient with continual disease activity developed gestational hypertension. The other UC patients had no pregnancy complications. The newborns of UC patients had Apgar score over 5 points and none of them had serious complications or malformations. Babies of two UC patients with mild disease activity developed physiological jaundice and the jaundice disappeared gradually without intensive care. There was no difference in newborn outcomes, pregnancy complications, or modes of delivery between the $\mathrm{CD}$ and UC patients who gave birth after confirmation of IBD $(\mathrm{P}>0.05)$ (Table 4).

Patients who were included from our hospital and who had been pregnant before confirmation of IBD diagnosis were asked to provide fertility information in an effort to determine whether there would be a difference in pregnancy outcomes between the patients who became pregnant before the IBD diagnosis and the patients who became pregnant after the diagnosis. Results revealed that there was no significant difference $(P>0.05)$ (Table 5). We then conducted a multivariate analysis that included age and disease course. However, there was still no significant difference. 
Table 4. Fertility outcomes of women with IBD.

\begin{tabular}{ccc}
\hline & CD (n) & UC (n) \\
\hline Successfully delivered & 15 & 12 \\
Premature delivery & 0 & 0 \\
Mode of delivery & & 7 \\
Vaginal & 9 & 5 \\
Cesarean & 6 & \\
Pregnancy-related disease & & 1 \\
Yes & 0 & 11 \\
No & 15 & \\
Newborn outcomes & & 12 \\
Normal & 15 & 0 \\
Abnormal & 0 & 0 \\
SGA & 0 & 0 \\
Apgar $<5$ & 0 & 0 \\
Admission to NICU & 0 & \\
\hline
\end{tabular}

${ }^{\star}$ All P > 0.05. IBD, inflammatory bowel disease; CD, Crohn's disease; UC, ulcerative colitis; SGA, Small for gestational age defined as birth weight $<10$ th percentile; NICU, neonatal intensive care unit. Pregnancy-related disease include: miscarriage, premature delivery and preeclampsia.

Moreover, this study compared the pregnancy outcomes between patients with different disease activity during the pregnancy. However, we did not find any correlation between disease activity and poor pregnancy outcome $(\mathrm{P}>0.05)$. Regarding the mode of delivery, most $\mathrm{CD}$ patients with disease remission had a vaginal delivery $(6 / 9 ; 67 \%)$. However, patients with disease activity primarily had a cesarean delivery; none of them had a vaginal delivery, there was a statistically significant difference on the delivery method between patients with active disease and patients with disease remission $(\mathrm{P}=0.028)$. There was a trend for $\mathrm{CD}$ patients who had disease activity during pregnancy to be more likely to have a cesarean delivery. A similar observation was also made for the UC patients; $67 \%$ (4/6) of patients with disease remission had a vaginal delivery and $16.7 \%$ of patients with disease activity chose to have a cesarean delivery (Table 6).

\section{Discussion}

$\mathrm{CD}$ and UC are both chronic inflammatory diseases. Recently, their incidence has increased in the Chinese population [1]. Most patients were of childbearing age when diagnosed, and many of them had not given birth but had the desire to do so. Therefore, it is of great importance to determine whether IBD and its medications would have an adverse impact on the fertility of these women. There have been no relevant reports regarding this matter in the Chinese population until now. We conducted a retrospective survey of pregnancy outcomes for women of childbearing age with IBD and performed statistical analysis.

A total of 139 (82 CD and 57 UC) patients were included in this study, including $90(64.7 \%)$ patients who chose not to purse pregnancy after the confirmation of IBD. All 90 patients were surveyed by one of two IBD clinics about the 
Table 5. Comparison of fertility outcomes for IBD patients before and after confirmation of IBD (single-center data).

\begin{tabular}{|c|c|c|c|c|}
\hline & \multicolumn{2}{|c|}{$\begin{array}{l}\text { Comparison of fertility } \\
\text { outcomes before and after the } \\
\text { confirmation of } C D\end{array}$} & \multicolumn{2}{|c|}{$\begin{array}{l}\text { Comparison of fertility } \\
\text { outcome before and after } \\
\text { confirmation of UC }\end{array}$} \\
\hline & $\begin{array}{l}\text { Fertility } \\
\text { outcomes after } \\
\text { diagnosis } \\
\text { confirmation }\end{array}$ & $\begin{array}{l}\text { Fertility } \\
\text { outcomes } \\
\text { before } \\
\text { diagnosis } \\
\text { confirmation }\end{array}$ & $\begin{array}{l}\text { Fertility } \\
\text { outcomes } \\
\text { after } \\
\text { diagnosis } \\
\text { confirmation }\end{array}$ & $\begin{array}{l}\text { Fertility } \\
\text { outcomes } \\
\text { before } \\
\text { diagnosis } \\
\text { confirmation }\end{array}$ \\
\hline $\begin{array}{c}\text { Prior } \\
\text { childbirth }\end{array}$ & 4 & 19 & 9 & 22 \\
\hline Premature delivery & 0 & 1 & 1 & 0 \\
\hline \multicolumn{5}{|l|}{ Mode of delivery } \\
\hline Vaginal delivery & 4 & 13 & 4 & 17 \\
\hline Cesarean delivery & 0 & 6 & 5 & 5 \\
\hline \multicolumn{5}{|l|}{$\begin{array}{c}\text { Pregnancy-related } \\
\text { disease }\end{array}$} \\
\hline Yes & 0 & 0 & 1 & 0 \\
\hline No & 4 & 19 & 8 & 0 \\
\hline \multicolumn{5}{|l|}{$\begin{array}{l}\text { Newborn } \\
\text { outcomes }\end{array}$} \\
\hline Normal & 4 & 19 & 9 & 0 \\
\hline Abnormal & 0 & 0 & 0 & 0 \\
\hline SGA & 0 & 0 & 0 & 0 \\
\hline Apgar $<5$ & 0 & 0 & 0 & 0 \\
\hline $\begin{array}{l}\text { Admission to } \\
\text { NICU }\end{array}$ & 0 & 0 & 0 & 0 \\
\hline
\end{tabular}

*All P > 0.05. IBD, inflammatory bowel disease; CD, Crohn's disease; UC, ulcerative colitis; SGA, Small for gestational age defined as birth weight $<10$ th percentile; NICU, neonatal intensive care unit. Pregnancy-related disease include: miscarriage, premature delivery and preeclampsia.

Table 6. Effects of disease activity during pregnancy on the mode of delivery.

\begin{tabular}{cccc}
\hline & Remission period & Active period & P-value \\
\hline CD & & & \\
Mode of delivery & 6 & 0 & 0.028 \\
Vaginal delivery & 3 & 6 & \\
Cesarean delivery & & & \\
UC & 4 & 1 & 0.242 \\
Mode of delivery & 2 & 5 & \\
Vaginal delivery & & & \\
Cesarean delivery & & &
\end{tabular}

reasons for not pursuing pregnancy, with $76.3 \%$ of the patients indicating that the reason was the national family planning policy and $23.7 \%$ indicating that the reason was concern about the adverse effects of disease activity, disease heredity, 
and medication on the fetus. The findings of this study were in agreement with those reported by other countries. For example, a study of the European and American population indicated that $18 \%$ of CD patients and $14 \%$ of UC patients opted to not pursue pregnancy, whereas only $6.2 \%$ of those without IBD opted to not pursue pregnancy; therefore, the rate of IBD patients not pursuing pregnancy was significantly higher than that of those without IBD $(\mathrm{P}<0.05)[10]$. A study performed in Australia stated that approximately $42.7 \%$ of IBD patients were concerned about the adverse effects on their fertility or delivery; however, they were shown to have fertility rates that were similar to those of the normal population ( $\mathrm{P}=0.553$ ), with no evidence-based medicine supporting the concern that disease heredity or medication would have adverse effects on pregnancy outcomes [11]. IBD has imposed a severe mental burden on women of childbearing age with IBD in China as well as in Europe and the United States, and this has led to many women deciding not to pursue pregnancy. Clinical physicians should place a higher emphasis on the education of women regarding pregnancy and IBD to help them make fertility decisions that are right for them and to prevent patients from not pursuing pregnancy because of their misconceptions.

In this study, except for two patients with UC, the women had been subjected to drug maintenance treatment prior to pregnancy, including the use of immunosuppressants and biological agents. Fifteen $(15 / 25,60 \%)$ patients discontinued all drugs for 3 months prior to conception. Ten patients continued to use oral mesalazine and one patient continued using azathioprine during pregnancy. Most of the patients had disease remission. Four CD patients had mild disease activity, including two patients who continued to have active disease during pregnancy but refused any medication. Five UC patients had mild disease activity when pregnant but refused to use any drugs other than mesalazine. During pregnancy, two CD patients with disease remission developed mild recurrence of the disease but refused any medication. One UC patient developed mild disease recurrence and agreed to use only mesalazine treatment. Women with IBD in China usually choose to give birth during disease remission with the guidance of physicians, and such patients included in this study did not have poor pregnancy outcomes, which was consistent with reports from other countries indicating that women with IBD with disease remission have pregnancy outcomes that are similar to those of the population without IBD [12] [13]. However, during disease activity, women with IBD in this study refused to use any IBD drug therapy other than mesalazine. This was attributed to the fact that many patients were worried about the adverse effects of the medication on pregnancy, such as the teratogenic effects of thalidomide on the fetus. The selective administration of drugs during pregnancy is still controversial, but studies performed in countries other than China have revealed that clinical physicians and patients usually overestimate the side effects of drug administration during pregnancy [14] [15]. Many studies have suggested that disease activity would increase poor pregnancy 
outcomes; therefore, it is important to achieve and maintain disease remission before conception and to undergo maintenance treatment during pregnancy [5] [16]. In the presence of disease activity during pregnancy, medication is considered the best option for pregnant women and fetuses. In such a scenario, the side effects of the medication are considered less important. A consensus published by $\mathrm{CD}$ and colitis organizations in Europe indicated that mesalazine and biological agents considered pregnancy class B drugs are safe to use during pregnancy [17]. A study also reported that the children whose mothers had been exposed to infliximab during pregnancy had growth rates and brain development that were similar to those of normal children, as observed in a long-term follow-up of these children [18]. Another study conducted a 6-year follow-up of the children whose mothers received azathioprine during their pregnancy and found that there was no significant difference in the immunologic functions or growth and development of those children compared with other children whose mothers did not use azathioprine [19]. Therefore, the adverse impact of IBD medication on pregnancy has been overestimated, and the side effects may be less emphasized.

In this study, nine CD patients were always in a state of disease remission before and during the pregnancy, and six CD patients presented with disease activity before or during the pregnancy. Of the UC patients, six were always in a state of disease remission and six presented with mild disease activity during the pregnancy. There was no significant difference in the poor pregnancy outcomes of patients with disease remission compared with those with active disease. Pregnancy outcomes were not significantly different before and after the onset of disease in this study $(\mathrm{P}>0.05)$, and the results were similar to those of case-control studies conducted in the European and American populations that reported that the pregnancy outcomes of women with IBD women were similar to those of women without IBD [20] [21]. However, disease activity during pregnancy is considered a major factor leading to aggravation of the disease and pregnancy complications [22] [23]. A meta-analysis suggested that although most women with IBD had normal pregnancy outcomes, they are at higher risk for premature delivery, having low-birth-weight infants, and requiring cesarean delivery compared to women without IBD [5]. This study further compared the effects of disease activity on the mode of delivery for the patients. It was found that IBD patients during a period of disease activity tended to be more likely to have a cesarean delivery, especially in CD patients. Because of the limited number of cases included in this retrospective study, a correlation of IBD and its activity with poor pregnancy outcomes was not found. A prospective, multicenter study with large samples is required to gain deeper insights regarding this issue.

Our study had some limitations. Firstly, the estimated sample size of the study was very large; therefore we enrolled patients in two large IBD centers to get as many patients as possible. However, the actual sample size was still not large enough which is partly due to the low incidence of IBD in Chinese females. The 
relatively small sample size may have had an influence on the study results. In addition, some patients refused to take part in our study because of personal privacy concerns, which further reduced the number of patients included. The relatively small sample size may have had an influence on the study results. Secondly, as a retrospective study, patients' information was collected by telephone interview which may had an influence on the reality of the answers and the investigation may be not comprehensive enough. Mailing the questionnaires to patients may be a better design and could improve the response rate and the reality of the answers. In totally, a large multi-center study with a larger sample size and better design is warranted to confirm the findings of this study. Firstly, the estimated sample size of the study was very large; therefore we enrolled patients in two large IBD centers to get as many patients as possible. However, the actual sample size was still not large enough which is partly due to the low incidence of IBD in Chinese females. In addition, some patients refused to take part in our study because of personal privacy concerns, which further reduced the number of patients included. The relatively small sample size may have had an influence on the study results. Secondly, as a retrospective study, patients' information was collected by telephone interview which may also had an influence on the reality of the answers and the investigation may be not comprehensive enough. Mailing the questionnaires to patients may be a better design and could improve the response rate and the reality of the answers. In totally, a large multi-center study with a larger sample size and better design is warranted to confirm the findings of this study.

This study suggested that many women with IBD may choose not to pursue pregnancy because of their concerns about the adverse effects of IBD or its medication on the pregnancy. Therefore, clinical physicians should educate patients about the relevant knowledge regarding the effects of IBD heredity, medication safety, and disease activity to reduce the number of patients who unnecessarily choose not to pursue pregnancy because of their misconceptions. In this study, disease activity was not found to have an obvious adverse effect on the pregnancy outcomes of IBD patients except the delivery mode. Women with IBD in China usually choose to pursue childbearing during the disease remission period, and most of the patients refused any IBD medication other than mesalazine. Moreover, the recurrence of disease during pregnancy is usually mild and not very frequent. Considering the low incidence of IBD in China and the limited number of samples included in this study, a long-term follow-up study including large samples is required to further confirm the relationship between IBD and pregnancy outcomes.

\section{Acknowledgements}

Professor Xiang Gao contributed to the design of the study and conducting the research comprehensively. Doctor Miao Li and Qingfan Yang were responsible for following up patients, statistical analysis and drafting the article. Doctor Jian 
Tang, and kang Chao helped colleting part of the patients' data. Doctor Lingjie Huang and Yuan Zhao helped collecting the control's data. Professor Min Zhi helped data acquisition. Professor Qian Cao contributed to the analysis of data. Professor Pinjin Hu supervised the research and revised the article.

\section{Conflicts of Interest and Source of Funding}

To the best of our knowledge, no conflict of interest, financial or other, exists. This work was supported by National Key Clinical Discipline, the National Nature Science Fund of China (No. 81370498).

\section{References}

[1] Zeng, Z., Zhu, Z., Yang, Y., Ruan, W., Peng, X., Su, Y., et al. (2013) Incidence and Clinical Characteristics of Inflammatory Bowel Disease in a Developed Region of Guangdong Province, China: A Prospective Population-Based Study. Journal of Gastroenterology and Hepatology, 28, 1148-1153. https://doi.org/10.1111/jgh.12164

[2] Riis, L., Vind, I., Politi, P., Wolters, F., Vermeire, S., Tsianos, E., et al. (2006) Does Pregnancy Change the Disease Course? A Study in a European Cohort of Patients with Inflammatory Bowel Disease. The American Journal of Gastroenterology, 101, 1539-1545. https://doi.org/10.1111/j.1572-0241.2006.00602.x

[3] Baird, D.D., Narendranathan, M. and Sandler, R.S. (1990) Increased Risk of Preterm Birth for Women with Inflammatory Bowel Disease. Gastroenterology, 99, 987-994. https://doi.org/10.1016/0016-5085(90)90617-A

[4] Bortoli, A., Pedersen, N., Duricova, D., D’Inca, R., Gionchetti, P., Panelli, M.R., et al. (2011) Pregnancy Outcome in Inflammatory Bowel Disease: Prospective European Case-Control ECCO-EpiCom Study, 2003-2006. Alimentary Pharmacology \& Therapeutics, 34, 724-734. https://doi.org/10.1111/j.1365-2036.2011.04794.x

[5] Cornish, J., Tan, E., Teare, J., Teoh, T.G., Rai, R., Clark, S.K., et al. (2007) A Meta-Analysis on the Influence of Inflammatory Bowel Disease on Pregnancy. Gut, 56, 830-837.

[6] Oron, G., Yogev, Y., Shcolnick, S., Hod, M., Fraser, G., Wiznitzer, A., et al. (2012) Inflammatory Bowel Disease: Risk Factors for Adverse Pregnancy Outcome and the Impact of Maternal Weight Gain. The Journal of Maternal-Fetal \& Neonatal Medicine, 25, 2256-2260. https://doi.org/10.3109/14767058.2012.684176

[7] Lennard-Jones, J.E. (1989) Classification of Inflammatory Bowel Disease. Scandinavian Journal of Gastroenterology, 170, 2-6.

https://doi.org/10.3109/00365528909091339

[8] Silverberg, M.S., Satsangi, J., Ahmad, T., Arnott, I.D., Bernstein, C.N., Brant, S.R., et al. (2005) Toward an Integrated Clinical, Molecular and Serological Classification of Inflammatory Bowel Disease: Report of a Working Party of the 2005 Montreal World Congress of Gastroenterology. Canadian Journal of Gastroenterology, 19, 5A-36A.

[9] Dollberg, S., Haklai, Z., Mimouni, F.B., Gorfein, I. and Gordon, E.S. (2005) Birth Weight Standards in the Live-Born Population in Israel. Israel Medical Association Journal, 7, 311-314.

[10] Marri, S.R., Ahn, C. and Buchman, A.L. (2007) Voluntary Childlessness Is Increased in Women with Inflammatory Bowel Disease. Inflammatory Bowel Diseases, 13, 591-599. https://doi.org/10.1002/ibd.20082 
[11] Mountifield, R., Bampton, P., Prosser, R., Muller, K. and Andrews, J.M. (2009) Fear and Fertility in Inflammatory Bowel Disease: A Mismatch of Perception and Reality Affects Family Planning Decisions. Inflammatory Bowel Diseases, 15, 720-725. https://doi.org/10.1002/ibd.20839

[12] Reddy, D., Murphy, S.J., Kane, S.V., Present, D.H. and Kornbluth, A.A. (2008) Relapses of Inflammatory Bowel Disease during Pregnancy: In-Hospital Management and Birth Outcomes. The American Journal of Gastroenterology, 103, 1203-1209.

[13] Stephansson, O., Larsson, H., Pedersen, L., Kieler, H., Granath, F., Ludvigsson, J.F., et al. (2011) Congenital Abnormalities and Other Birth Outcomes in Children Born to Women with Ulcerative Colitis in Denmark and Sweden. Inflammatory Bowel Diseases, 17, 795-801. https://doi.org/10.1002/ibd.21369

[14] Sanz, E., Gomez-Lopez, T. and Martinez-Quintas, M.J. (2001) Perception of Teratogenic Risk of Common Medicines. European Journal of Obstetrics and Gynecology, 95, 127-131. https://doi.org/10.1016/S0301-2115(00)00375-4

[15] Tomson, T., Battino, D., Bonizzoni, E., Craig, J., Lindhout, D., Sabers, A., et al. (2011) Dose-Dependent Risk of Malformations with Antiepileptic Drugs: An Analysis of Data from the EURAP Epilepsy and Pregnancy Registry. The Lancet Neurology, 10, 609-617. https://doi.org/10.1016/S1474-4422(11)70107-7

[16] Nguyen, G.C., Boudreau, H., Harris, M.L. and Maxwell, C.V. (2009) Outcomes of Obstetric Hospitalizations among Women with Inflammatory Bowel Disease in the United States. Clinical Gastroenterology and Hepatology, 7, 329-334. https://doi.org/10.1016/j.cgh.2008.10.022

[17] Van der Woude, C.J., Kolacek, S., Dotan, I., Oresland, T., Vermeire, S., Munkholm, P., et al. (2010) European Evidenced-Based Consensus on Reproduction in Inflammatory Bowel Disease. Journal of Crohn's and Colitis, 4, 493-510. https://doi.org/10.1016/j.crohns.2010.07.004

[18] Bortlik, M., Duricova, D., Machkova, N., Kozeluhova, J., Kohout, P., Hrdlicka, L., et al. (2014) Impact of Anti-Tumor Necrosis Factor Alpha Antibodies Administered to Pregnant Women with Inflammatory Bowel Disease on Long-Term Outcome of Exposed Children. Inflammatory Bowel Diseases, 20, 495-501. https://doi.org/10.1097/01.MIB.0000440984.86659.4f

[19] De Meij, T.G., Jharap, B., Kneepkens, C.M., van Bodegraven, A.A. and de Boer, N.K. (2013) Dutch Initiative on Crohn and Colitis. Long-Term Follow-Up of Children Exposed Intrauterine to Maternal Thiopurine Therapy during Pregnancy in Females with Inflammatory Bowel Disease. Alimentary Pharmacology \& Therapeutics, 38, 38-43. https://doi.org/10.1111/apt.12334

[20] Willoughby, C.P. and Truelove, S.C. (1980) Ulcerative Colitis and Pregnancy. Gut, 21, 469-474. https://doi.org/10.1136/gut.21.6.469

[21] Khosla, R., Willoughby, C.P. and Jewell, D.P. (1984) Crohn's Disease and Pregnancy. Gut, 25, 52-56. https://doi.org/10.1136/gut.25.1.52

[22] Fedorkow, D.M., Persaud, D. and Nimrod, C.A. (1989) Inflammatory Bowel Disease: A Controlled Study of Late Pregnancy Outcome. Am J Obstet Gynecol, 160, 998-1001. https://doi.org/10.1016/0002-9378(89)90324-4

[23] Nørgård, B., Pedersen, L., Christensen, L.A. and Sørensen, H.T. (2007) Therapeutic Drug Use in Women with Crohn's Disease and Birth Outcomes: A Danish Nationwide Cohort Study. The American Journal of Gastroenterology, 102, 1406-1413. https://doi.org/10.1111/j.1572-0241.2007.01216.x 